\title{
Three-Dimensional Device Fabrication in Monocrystalline Diamond Using FIB and a Novel Lift-off Technique
}

\author{
P. Olivero, ${ }^{*}$ S. Rubanov, * P. Reichart,* S. Huntington,* B. Gibson,* J. Salzman, ** S. Prawer, ${ }^{*}$ D. N. \\ Jamieson* \\ *School of Physics, Microanalytical Research Centre, University of Melbourne, Victoria, 3010, \\ Australia \\ ** Department of Electrical Engineering, Technion, Israel Institute of Technology, Haifa 3200, Israel
}

Diamond has extreme properties, such as the highest mechanical hardness and Young modulus, chemical inertness, high thermal conductivity, high refractive index, optical transparency, and a series of well-characterized photoluminescent centers. These physical properties offer a wide range of applications for diamond microstructures in integrated micro-optics, micro-electromechanical systems (MEMS) technology and microfluidics [1-2]. However, because of these extreme properties the device fabrication in single crystal diamond is very difficult. Focused ion beam (FIB) technique is probably only method which can be used for micromachinig of crystalline diamond with submicron resolution.

In this work we present a novel FIB assisted lift-off technique for three-dimensional device fabrication in bulk single-crystal diamond. The method involves $2 \mathrm{MeV} \mathrm{He}$ ion implantation to produce a buried damaged layer, thermal annealing for its graphitization, followed by pattern milling with a focused $30 \mathrm{keV} \mathrm{Ga}$ ion beam and chemical etching of the patterned regions. The chemical etching take place only in the buried graphite layer in the areas where it was exposed by FIB patterns and FIB patterned regions are lifted off. Free-standing three-dimensional structures with well defined micrometric features are obtained. The thickness and the depth of the buried graphite layer can be modified by variation of ion dose and energy during He ion implantation.

Using this technique we fabricated diamond cantilever (Fig.1a) and diamond bridge-like structure for optical waveguiding (Fig.1b). In order to test possible applications of the bridge-like structures in integrated optics, the FIB patterning of the bridge-like structure was integrated with an input/output light collection system based on linear FIB milled trenches inclined at $45^{\circ}$ with respect to the sample surface, at the two ends of the structure. Their function of mirrors is based on the internal reflection in diamond with respect to the air. The refractive index of diamond is $n=2.41$, which determines a very small critical angle $\left(\theta_{c} \approx 25^{\circ}\right) .533 \mathrm{~nm}$ laser light was focused on a micrometer spot at one of the two mirrors, and the same focusing optics were used to collect the light coming out of the second mirror. The bridge-like microstructure acts as an effective waveguide, confining the propagating light and exhibiting a structured pattern at the output spot (Fig.2), which is indicative of the reciprocal interference of the many modes propagating along the $\sim 2.5 \times 3.5 \mu \mathrm{m}^{2}$ section of the structure.

\section{References}

[1] M. Y. Mao, T. P. Wang, J. F. Xie, W. Y. Wang, Proceedings IEEE Micro Electro Mechanical Systems, Amsterdam, Netherlands (1995) 392.

[2] H. Björkman, P. Rangsten, P. Hollman, K. Hjort, Sensors and Actuators 73 (1999) 20. 

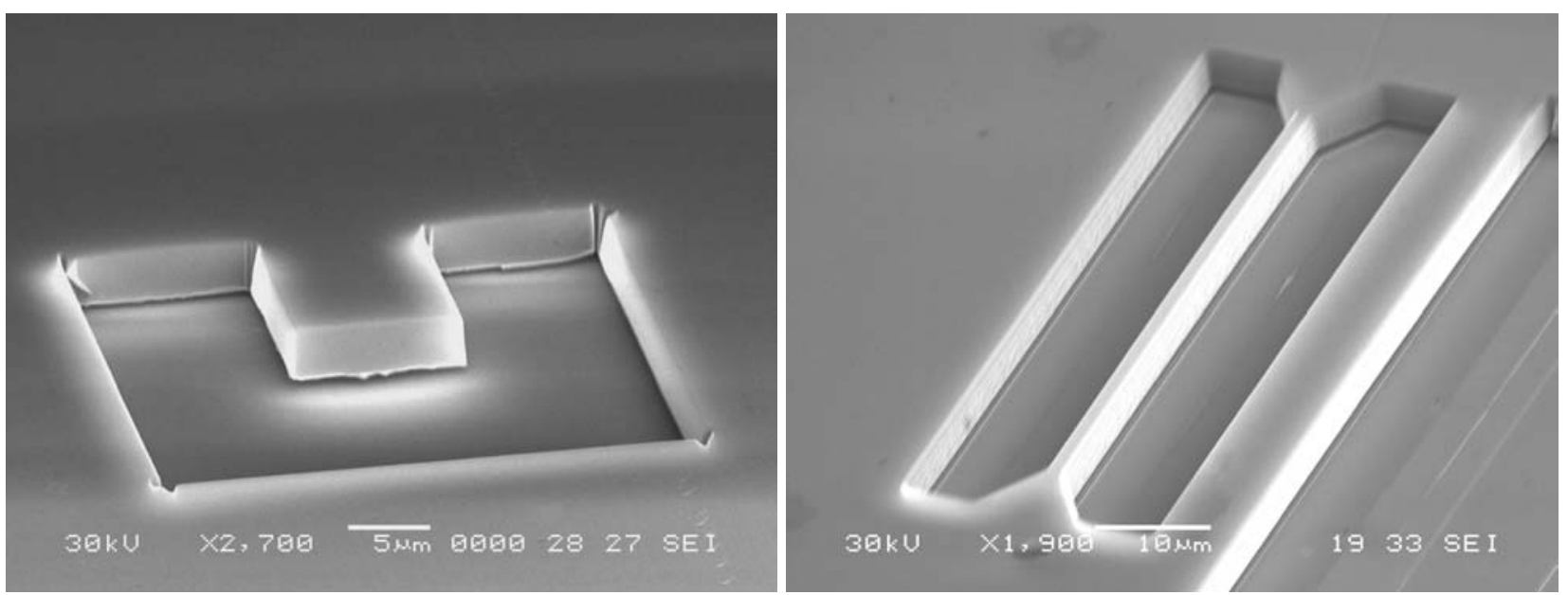

Fig. 1.SEM images of three-dimensional structures fabricated in single-crystalline diamond. A. Cantilever. B. The bridge-like structure for optical waveguide.

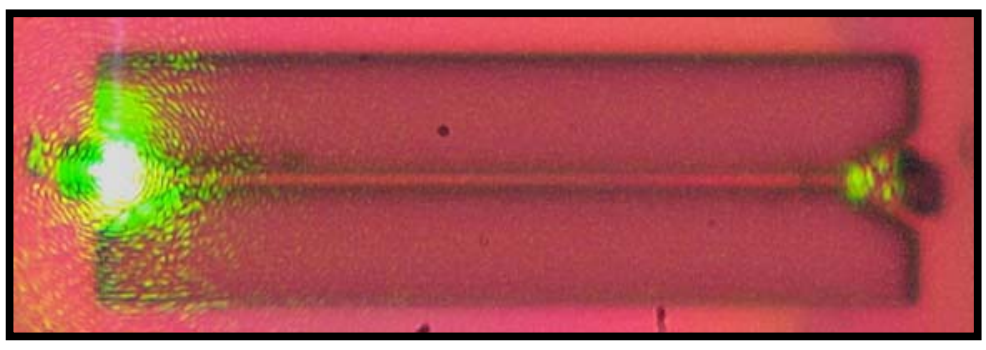

2. Optical image of working waveguide. 